\title{
Arthroscopic Popliteus sling Reconstruction using the 'Popliteus portal' : An effective way to restore the Posterolateral corner stability A Case Series and Surgical Technique
}

Abey Thomas Babu ( $\sim$ abey.babu@gmail.com )

Ortho One Orthopaedic Speciality Centre

Santosh Sahanand

Ortho One, Orthopaedic Speciality Centre, Coimbatore

David Rajan

Ortho One Orthopaedic Speciality Centre, Coimbatore

Research article

Keywords: Knee, Posterolateral corner injury, popliteus, Arthroscopy, Reconstruction

Posted Date: September 5th, 2019

DOI: https://doi.org/10.21203/rs.2.13915/v1

License: (c) (1) This work is licensed under a Creative Commons Attribution 4.0 International License.

Read Full License 


\section{Abstract}

Background: Posterolateral corner injuries can result in persistent varus and rotary instability. Many open/ arthroscopic procedures of reconstruction/ repair have been reported, but there is a paucity of literature on clinical outcomes. We follow an all arthroscopic reconstruction technique of the popliteus sling with the use of the 'popliteus portal' in cases of isolated popliteus injuries (intact fibular collateral ligament). Methodds: Prospective case study of 12 patients undergoing Arthroscopic Popliteus sling reconstruction with or without associated cruciate ligament reconstruction was peformed. We report our surgical technique and clinical outcomes. Results: All our patients had good to excellent knee function at final follow up (IKDC and Tegner Scores). We did not encounter any major complications intra or post operatively. Conclusions: In cases of Popliteus tendon injury without fibular collateral ligament injury, an 'all - arthroscopic' Popliteus sling reconstruction is an effective and reproducible technique of restoring posterolateral stability of the knee. The advantages of our procedure are - an 'all - arthroscopic Technique', avoiding damage to the meniscotibial ligaments and a more 'anatomic' reconstruction of the popliteus sling. Keywords: Knee, Posterolateral corner injury, popliteus, Arthroscopy, Reconstruction

\section{Background}

The posterolateral corner [PLC] of the knee consists of an array of complex ligamentous and musculotendinous structures and is an essential element for a functional lower extremity. The primary function of the PLC is to resist varus, external rotation and posterior translation of the tibia. A neglected or undiagnosed PLC injury can lead to significant disability and affect activities of daily living, recreational and sporting activities. A thorough understanding of the complex anatomy and biomechanics of the PLC will help the Orthopaedic surgeon in this challenging diagnostic and therapeutic scenario. ${ }^{1}$

The awareness of neglected Posterolateral corner injuries as a cause of cruciate ligament reconstruction failures is on the rise. The popliteus is an important rotary stabilizer of the posterolateral complex. ${ }^{2}$

Anatomically the 3 primary structures contributing to the static stability of the PLC are the fibular collateral ligament [FCL], the popliteus tendon [PLT] and the popliteofibular ligament [PFL\} A high index of suspicion, a thorough physical examination along with a comprehensive review of radiographic and Magnetic Resonance Imaging [MRI] is needed for an accurate diagnosis of PLC injuries. The clinical tests for diagnosing PLC injuires are reverse pivot shift test, dial test, varus stress test, hughston test, posterolateral drawers test and the varus recurvatum test. ${ }^{2,3}$

Common mechanisms of injury include a posterolaterally directed blow to the anteromedial proximal tibia, with resultant hyperextension; a noncontact hyperextension and external rotation twisting injury; direct blow to a flexed knee; or high energy trauma. Injuries to the PLC are often combined with other ligamentous injuries, especially those of the Posterior Cruciate ligament [PCL]. Injury to the popliteofibular ligament complex or avulsions and tears of the popliteus, primarily result in abnormal posterolateral rotatory laxity. Injury to the fibular collateral ligament primarily leads to varus laxity. ${ }^{4}$ 
Multiple techniques of repair and reconstruction of the posterolateral corner have been described in literature, using open and arthroscopic methods. Primary repair of PLC has been reported to yield good results, if performed within 3 weeks. PLC reconstructions can be broadly divided into two categories: nonanatomic and anatomic. Anatomic reconstruction can be broadly separated into fibular-based and combined tibial-fibular-based reconstructions. ${ }^{5}$

We follow an all arthroscopic anatomic tibial-based reconstruction technique of the popliteus sling with the use of the 'popliteus portal' in cases of isolated PLT injuries(FCL component intact), which is minimally invasive, more anatomic, cosmetic, with better rehabilitation and providing rapid recovery than an open procedure. The other described technique in literature involves the use of a 'posterolateral portal' which involves dissection of the meniscotibial ligaments, which is also a stabiliser of the posterolateral corner.

We report our surgical technique and outcomes of 12 cases of all arthroscopic reconstruction of the popliteus tendon using the 'popliteus portal' as described by Kodkani. ${ }^{2}$

\section{Methods}

We present a case series of patients undergoing Arthroscopic Popliteus sling reconstruction with or without associated cruciate ligament reconstruction at our institution between January 2016 and December 2018. The aim of our study was to evaluate the efficacy of arthroscopic Popliteus sling reconstruction in restoring posterolateral corner stability. The study was approved by our institutional review board, and informed patient consent for study inclusion was obtained.

The inclusion criteria were patients with popliteus tendon injury with posterolateral rotary instability as identified by a positive hughston test without FCL injury [varus instability]. Associated cruciate ligament injuries or meniscal injuries were not grounds for exclusion.

Patients who had FCL injury and varus instability were excluded as they were treated with an open FCL repair [in acute setting] or an open FCL reconstruction [Modified Larson] technique. Cases with fractures of the proximal tibia or distal femur, open injury, or associated neurovascular injury were excluded from the study.

Clinically, each patient was assessed preoperatively and under anesthesia using the Lachman and anterior and posterior drawer tests to evaluate for cruciate injury, as well as varus and valgus stress tests both in extension and at $30^{\circ}$ of flexion to assess the competence of the collateral ligaments. The dial, external rotation recurvatum and hughstons tests were also performed to evaluate posterolateral corner injuries.

Stress Radiographs were used to evaluate the posterior sag in cases with PCL injuries. MRI scan was done for all patients to supplement the clinical tests in arriving at an accurate diagnosis. 
Patients were followed up at 6 weeks, 3, 6, 12 and 18 months post operatively. The parameters assessed during follow up were the IKDC and Tegner score, the ROM, posterior sag and Hughston test for posterolateral laxity.

\section{Surgical Technique}

In the operating room, the injured knee was examined with the patient under anesthesia and laxity elicited was documented. The patient is kept in supine position under spinal anesthesia with the limb secured in a leg holder ensuring that the knee is able to hang up to at least 90 degrees of flexion. The procedure is performed under tourniquet control after exsanguination of the limb. A standard 30 degrees arthroscope is used. Initially a diagnostic arthroscopy is performed to identify associated cruciate/ meniscal injuries using the standard anteromedial and anterolateral portals.

\section{Popliteus Portal}

Under direct vision 'the popliteus portal' is made using the outside in technique centered over the popliteus femoral footprint at 70 degree of knee flexion. (Fig 1) This portal passes through the skin, subcutaneous tissue, iliotibial tract, capsule, and the synovium. It does not injure any significant neurovascular structure. The portal should be directed in a way which will permit the reaming of femoral tunnel for the graft in the popliteus groove in the lateral condyle of femur, directed in a proximal and anterior direction.

\section{Graft harvesting and preparation}

Autologous gracilis tendon from the ipsilateral knee was used for reconstruction of the popliteus sling. In cases with multiligament injury, where graft could not be acquired from the ipsilateral knee, contralateral knee gracilis was harvested for the graft. A minimum of $180-200 \mathrm{~mm}$ of graft was required for the Popliteus sling reconstruction. After clearing the muscle fibers, the ends of the graft were whipstitched with no.2 fibrewire/ Ethibond for a length of $30 \mathrm{~mm}$ on either ends.

\section{Tibial tunnel}

This bone tunnel is made anteroposteriorly, starting in the distal portion of flat triangular area in between the Gerdy tubercle and the lateral border of patellar tendon insertion. The point of exit for the tunnel is just below the ridge on the posterior aspect of the lateral tibial plateau where it dips into a sulcus, usually at the level of the popliteus musculotendinous junction. The point of exit should also be medial in relation to the lateral wall of the lateral femoral condyle. The tunnel is therefore drilled in a posterolateral direction to avoid it from being directly in line with the neurovascular structures behind.(Fig 2)

An anterior cruciate ligament elbow aimer (Acufex) is used for making this tunnel. (Fig 3)The tip of the aimer is passed through the popliteus portal across the popliteal hiatus (viewing portal being the anterolateral portal)and then onto the posterior aspect of the tibia. The tip of the aimer is used to palpate the popliteus ridge posteriorly and then moved further distally till it dipped into the sulcus. One may need 
to flex the knee up to $20-30$ degrees in this step for better visualization. A beath pin is then passed through till it is visualized exiting at the elbow of the aimer. The aimer is removed and the tip of the pin protected by a small curvedscoop passed through the popliteus portal to prevent it from advancing further while reaming. A $6 \mathrm{~mm}$ reamer is used. The tunnel is reamed cautiously to avoid a sudden exit from the posterior aspect. The reaming is stopped as soon as the tip of the reamer is visualized. The exit point could be cleared with a shaver passed through the bone tunnel or the popliteus portal.

A suture loop of no.1 prolene mounted on a knot pusher is used to serve as a shuttle for passing the graft. It is inserted through the tunnel advancing the knot pusher and the tip of the prolene loop till it can be visualized exiting posteriorly. A suture retriever is passed through the popliteus portal across the popliteal hiatus to withdraw the prolene suture into the lateral gutter and then further out of the popliteus portal.

\section{Femoral Tunnel}

The site for the tunnel is at the anterior end of anatomic insertion site of the popliteus. The insertion site is at the anterior end of the popliteal groove on the femoral condyle, close to where the articular cartilage ends approximately $2 \mathrm{cmm}$ distal and anterior to the fibular collateral ligament insertion in line with the femoral axis. A guide pin is passed initially over which a $6 \mathrm{~mm}$ reamer is drilled.

The pin is directed proximally toward the region between the adductor tubercle and medial epicondyle and angulated anteriorly to exit through the skin in the region between the medial epicondyle and medial border of trochlea. This is to avoid tunneling into the intercondylar notch and having a more conducive angle for the graft at its entry into the tunnel.

Figures $4 \mathrm{a}$ and $4 \mathrm{~b}$ show a Diagramatic representation of Location of femoral and tibial tunnel for the popliteus graft in Anteroposterior and lateral views.

\section{Graft Passage and Fixation}

The graft is fed into the Prolene shuttle and pulled across the tibial tunnel through the popliteal hiatus and out of the popliteus portal. The Ethibond is then fed into the eyelet of the beath pin in the femoral tunnel and shuttled across along with the pin out of the skin on the medial side. The graft is pulled into the tunnel with the help of the Ethibond till it reaches the end of the femoral tunnel.

Femoral fixation is done using a biointerference screw $(7 \mathrm{~mm})$ while maintaining graft tension. Tibial fixation is also done using a bio interference screw $(7 \mathrm{~mm})$ keeping the knee at 90 degrees flexion correcting the poserolateral sag. This reduction is maintained by keeping the foot in 15 degrees internal rotation and maintaining graft tension while fixation of the graft.

Associated cruciate ligament reconstructions and meniscus surgery were performed following standard procedures. 


\section{Postoperative Rehabilitation}

The knee is kept non weight bearing for a period of 6 weeks after which partial weight bearing is permitted gradually to full weight bearing by 8 weeks. The knee is placed in a long knee brace in extension with posterior tibial support for a period of 3 weeks. Passive prone ROM is started at 3 weeks post op and the long knee brace is replaced with a ROM knee brace. Isometric quadriceps and short arc quadriceps exercises are started at 3 weeks postoperatively. Isotonic quadriceps, leg press, and gait training is started after 6 weeks. Proprioceptive training is started on regaining quadriceps strength equal to the opposite uninvolved knee. Hamstring strengthening exercises are delayed for 6 months

\section{Follow up Evaluation}

All the patients were followed up and evaluated by clinical and radiographic assessment at 6 weeks and $3,6,12,18$ months post operatively. The Tegner activity score and International Knee Documentation Committee (IKDC) knee ligament examination form were used for follow-up assessment to document Knee scores. In addition VAS score for pain and a subjective assessment of ROM and stability of the knee using the Posterior sag test and Hughston's test for posterolateral laxity were also performed and findings were documented.

\section{Statistical Analysis}

The data obtained was statistically analyzed with SPSS software (version 13.0; SPSS, Chicago, IL).

\section{Results}

A total of 12 cases of arthroscopic Popliteus sling reconsruction using the 'popliteus portal' were were included in this study per the inclusion criteria.(Fig 5)

All patients were males with a mean age of 33.7 years. Five patients were hyperlax with a Beighton score of $>4$. The mean time from injury to surgery was 115.6 weeks. $50 \%$ of the cases had sustained injury in a two - wheeler RTA, while $17 \%$ of the cases had a sports injury.(Fig 6)All patients had an associated PCL tear. The other associated injuries are summarised in table 1.

We got posterior manual stress radiographs to document the posterior sag for all patients. The average posterior sag was $15.4 \mathrm{~mm}$. Five of the patients had Grade II (Kellgren and Lawrence) OA changes on the pre - operative radiograph.

All patients underwent Poplitues sling reconstruction using the 'Popliteus portal' along with standard single bundle PCL reconstruction. The quadriceps tendon graft was used for PCL reconstruction in 10 patients and hamstring graft was used in 2 patients. The ipsilateral/ contralateral gracilis graft was used for Popliteus reconstruction in all patients $(n=12)$. The concomitant procedures are summarised in table 2. 


\section{Follow Up}

The mean follow up was 18 months (range 6-36 months)

\section{Tourniquet Time}

The average Tourniquet time improved from 127.5 minutes during $2106-2017$ to 120 minutes during 2018 , indicating a learning curve for the procedure. The difference was not statistically significant. Also because of the non - uniformity of the surgical procedures, this parameter may not be of great relevance.

\section{Range of Motion}

The average Pre-op ROM was 0 - 107 degrees which improved to 0 - 115 degrees at final follow up. (Fig 7)

Fig 8 shows the post - operative radiograph with the markings for the femoral and tibial tunnel for the popliteus reconstruction using the 'Popliteus Portal'.

\section{$\underline{\text { IKDC score }}$}

There was a significant improvement in the IKDC scores of all the patients at final follow up. ( $p$ value 0.002) (Fig 9)

\section{Tegner Activity Level}

The Tegner activity level improved at final follow up, however it was not statistically significant. (Fig 10)

\section{Posterior Sag/ Posterolateral Laxity.}

None of our patients had a significant posterior sag or posterolateral laxity (> Gr II Hughston's) at final follow up.

\section{Complications}

We did not encounter any major Intra op or Post op complications. Two of our patients had stiffness at 3 months follow up and one patient complained for persisting pain which responded to conservative measures. All our patients were asymptomatic at final follow up.

\section{Association of Other Variables}

Based on our analysis we identified factors associated with a better outcome which were age $<40$ years, absence of osteoarthritic changes and a time duration between injury and surgery of $<3$ months.

Hyperlaxity and concomitant surgical procedures did not adversely affect outcomes.

However, due to a small sample size no statistically significant associations could be drawn. 


\section{Discussion}

Clinically, injuries to the PLC or "dark side" of the knee are becoming more recognized. Failure on the part of the treating surgeon to identify and manage PLC injuries (mainly grade III injuries) may often lead to poor outcomes for the patient including failures of cruciate ligament reconstructions, chronic instability which may eventually result in early onset osteoarthritis, and chronic pain. In the recent past our understanding of the anatomy, biomechanics, diagnosis, and management of PLC injuries has increased and it is important for the sports medicine specialist to be aware of the latest trends..$^{3,6,7}$

Werner Muller in 1982 was the first to describe in literature, an anatomical reconstruction of the popliteus complex with an anatomical popliteus bypass graft. Thereafter, numerous surgical techniques to reconstruct the static stabilizing function of the PLT have been described. Most of these are extra/ or nonanatomical techniques which fail to completely stabilize the posterolateral corner. It is now believed that anatomical techniques for the reconstruction of the posterolateral corner render good and excellent results. However, the described techniques are basically open surgical procedures, without the advantages of an arthroscopic technique. ${ }^{4,7,8,9}$

Open reconstructions require a thorough knowledge of the anatomy of the lateral side of the knee, a rather large exposure of the posterolateral complex through a hockey stick incision, isolation of the common peroneal Nerve and is quite cumbersome. Despite the wide exposure, there is insufficient direct visualization of the deeper posterolateral structures and difficulty in having a complete intra-articular ligament construct for the popliteus. 2,10

The all arthroscopic technique using the "popliteus portal', described in this article helps in a more anatomic reconstruction of popliteus as it allows for visualisation and graft passage through the natural popliteal hiatus. It obviates the need for big incisions, wide exposure and results in least surgical trauma and negligible risk of complications, and therefore can be considered safe. This single portal provides for ease of access to the desired structures simplifying the procedure and the use of routine knee arthroscopy instrumentation makes it reproducible albeit with a learning curve. ${ }^{2}$

Frosch et al. described a technique of arthroscopic PLC stabilization with the help of a popliteus bypass graft , which is similar to our technique but involves the use of the posterolateral portal. They studied 35 patients who underwent the ' popliteus bypass' procedure for 1 year and reported no major complications. The mean Tegner Score was preoperatively $5.6( \pm 1.8)$ and $4.9( \pm 1.0)$ points during follow-up, which is comparable to our study. ${ }^{11}$

Hermanowicz et al. described an arthroscopic PLC stabilization technique using popliteus tenodesis with simultaneous preservation of the femoral attachment of PLT to restore the static function of popliteus complex with the help of a midlateral portal, which is similar to our 'popliteus portal. However, this technique cannot be applied when the femoral attachment of PLT is damaged or if the ligament is stretched out. ${ }^{12}$ 
Frings et al. in 2019 described an all-arthroscopic technique for complete PLC reconstruction, based on an open technique described by Arciero. This technique utilizes a fibular tunnel and two femoral tunnels for both the $\mathrm{FCl}$ and PLT components of PLC and requires a free graft. For dissecting the PLC region a posterolateral portal is used. ${ }^{10}$

These other technique for arthroscopic Popliteus reconstruction involves multiple portals - the anterolateral, posterolateral, superolateral, posteromedial, and the transseptal portal. It requires detaching/excision of a part of the posterolateral meniscocapsular ligaments which may add to the posterolateral laxity and also cause extravasation of saline into the posterior compartment and superficial planes. The visualization of the popliteus is from the extra-articular region making it less anatomical. In addition there is a risk of injury to the inferior lateral genicular vessels. Switching of portals multiple times for visualization, and instrumentation makes it technically difficult and eats into the precious tourniquet time as well.

All the above disadvantages are avoided by using the "popliteus portal" for reconstruction of the popliteus sling. The advantages of using a 'Popliteus portal' and our technique are summarised in table 3.

Most previous reports on posterolateral knee reconstructions were primarily descriptions of the techniques and provided few patient outcomes. However, no randomized controlled study has been carried out to compare its efficacy with any other similar procedure for management of posterolateral rotatory laxity. Our study is one of the first reported in the literature which studied patient outcomes at one year follow up after arthroscopic reconstruction of the popliteus sling.

The above procedure may be supplemented with a mini open fibular collateral ligament reconstruction for knees with concurrent abnormal varus laxity (grade III FCL laxity).

\section{Limitations}

The mean follow up period of our study group was $6-36$ months. Longer follow up is required before we can draw firm conclusions about the efficacy of this technique.

Isolated Popliteus tears (posterolateral laxity) is a rare clinical entity, which explains the few number of patients in our study. Due to the same reason statistical significance could not be attributed to most of the variables we have studied. Perhaps multicentre studies in the future may help us to study larger patient groups and obtain more meaningful results

All our patients had associated injuries in the form of PCL/ ACL or meniscus tears. Hence the surgical procedure, tourniquet time, graft choice etc lacked uniformity which may also have contributed a bias to the study.

\section{Conclusions}


In isolated cases of PCL with Popliteus tendon injury without FCL injury, an 'all - arthroscopic' Popliteus sling reconstruction is an effective and reproducible technique of restoring posterolateral instability of the knee. The advantages of our procedure are - an 'all - arthroscopic Technique', avoiding damage to the meniscotibial ligament and a more 'anatomic' reconstruction of the popliteus sling. There is a need for more randomised clinical studies to evaluate the outcomes and complications of the arthroscopic popliteus sling reconstruction technique. Only time and further research will tell us the effectiveness of this procedure.

\section{Abbreviations}

PLC - Posterolateral Corner

FCL - Fibular Collateral Ligament

PLT - Popliteus Tendon

PFL - Popliteofibular Ligament

MRI - Magnetic Resonance Imaging

PCL - Posterior Cruciate Ligament

$\mathrm{ACL}$ - Anterior Cruciate Ligament

IKDC - International Knee Documentation Committee

ROM - Range of Movements

VAS - Visual Analog Score

RTA - Road Traffic Accident

\section{Declarations}

\section{Ethics approval and consent to participate}

Ethics approval and consent was taken from the Institutional Human Ethics Committee (IHEC) PSGIMS\&R, PSG Hospitals, Coimbatore.

\section{Consent for publication}

Informed written Consent for publication was taken from all participants.

\section{Availability of data and materials}


All data generated or analysed during this study are included in this published article [and its supplementary information files].

\section{Competing interests}

The authors declare that they have no competing interests

\section{Funding}

The authors have not received any funding

\section{Authors' contributions}

SS and DVR operated on all patients. ATB analysed the patients, collected data and wrote the manuscript. All authors read and approved the final manuscript

\section{Acknowledgements}

Not Applicable

\section{References}

1. Sanchez AR 2nd, Sugalski MT, LaPrade RF. Anatomy and biomechanics of the lateral side of the knee. Sports Med Arthrosc Rev. 2006 Mar;14(1):2-11.

2. Kodkani P .Arthroscopic Popliteus Sling Reconstruction-The "Popliteus Portal". Techniques in Knee Surgery _ Volume 10, Number 1, March 2011.

3. Chahla J, Moatshe G, Dean CS, LaPrade RF. Posterolateral Corner of the Knee: Current Concepts. Arch Bone Jt Surg. 2016;4(2):97-103.

4. Ranawat A, Baker CL 3rd, Henry S, Harner CD. Posterolateral corner injury of the knee: evaluation and management. J Am Acad Orthop Surg.2008 Sep;16(9):506-18.

5. LaPrade RF, Johansen S, Agel J, Risberg MA, Moksnes H, Engebretsen L. Outcomes of an anatomic posterolateral knee reconstruction. J Bone Joint Surg Am.2010 Jan;92(1):16-22.

6. Geeslin AG, LaPrade RF. Outcomes of treatment of acute grade-III isolated and combined posterolateral knee injuries: a prospective case series and surgical technique. J Bone Joint Surg Am. 2011; 93(18):1672- 83.

7. LaPrade RF, Hamilton CD, Engebretsen L. Treatment or acute and chronic combined anterior cruciate ligament and posterolateral knee ligament injuries. Sports Med Arthrosc. 1997; 5(2):91-9. 
8. LaPrade RF, Johansen S, Wentorf FA, Engebretsen L, Esterberg JL, Tso A. An analysis of an anatomical posterolateral knee reconstruction: an in vitro biomechanical study and development of a surgical technique. Am J Sports Med.2004 Sep;32(6):1405-14.

9. Franciozi CE, Albertoni LJB, Gracitelli GC, et al. Anatomic Posterolateral Corner Reconstruction With Autografts. Arthrosc Tech. 2018;7(2):e89-e95. Published 2018 Jan 8. doi:10.1016/j.eats.2017.08.053

10. Frings, Jannik \& P. Kolb, Jan \& Drenck, Tobias \& Krause, Matthias \& Alm, Lena \& Akoto, Ralph \& Frosch, Karl-Heinz. (2019). Anatomic Reconstruction of the Posterolateral Corner: An All-Arthroscopic Technique. Arthroscopy Techniques. 8. 10.1016/j.eats.2018.10.010.

11. Frosch KH, Akoto R, Drenck T, Heitmann M, Pahl C, Preiss A. Arthroscopic popliteus bypass graft for posterolateral instabilities of the knee: A new surgical techniqueArthroskopisches "Popliteus Bypass Graft" für posterolaterale Rotationsinstabilitäten des Kniegelenks : Eine neue operative Technik. Oper Orthop Traumatol. 2015;28(3):193-203.

12. Hermanowicz K, Góralczyk A, Malinowski K, Jancewicz P. Arthroscopic Posterolateral Corner Stabilization With Popliteus Tenodesis. Arthrosc Tech. 2018;7(6):e669-e674.

\section{Tables}

A. Table 1 - Associated Injuries

\begin{tabular}{|c|c|}
\hline Associated Injuries & No of Patients \\
\hline PLT + PCL + MM tear & 8 \\
\hline$P L T+P C L+A C L+M M$ tear & 3 \\
\hline$P L T+P C L+A C L+M C L+M M$ tear & 1 \\
\hline
\end{tabular}

PLT - Popliteus tendon, PCL - Posterior Cruciate Ligament, MM - Medial Meniscus, ACL - Anterior Cruciate Ligament, MCL Medial Collateral Ligament

B. Table 2 - Surgical Procedure 


\begin{tabular}{|c|c|}
\hline Surgical Procedure & No of Patients \\
\hline PLTR + PCLR + Meniscus Sx & 8 \\
\hline PLTR + PCLR + ACLR + Microdrilling & 3 \\
\hline PLTR + PCLR + ACLR + MCLR & 1 \\
\hline
\end{tabular}

PLTR - Popliteus Reconstruction, PCLR - Posterior Cruciate Ligament Reconstruction, Sx - Surgery, ACLR - Anterior Cruciate Ligament Reconstruction, MCLR - Medial Collateral Ligament Reconstruction

C. Table 3 - Advantages of our technique

\begin{tabular}{|l|l|}
\hline & Advantages of 'Popliteus Portal' Technique \\
\hline 1. & Intra - articular visualisation and graft passage through the popliteal hiatus \\
2. & All arthroscopic technique \\
3. & Anatomic tunnel placement \\
4. & Avoid damage to meniscotibial ligaments \\
5. & No dissection of Common Peroneal Nerve \\
6. & Safe and reproducible procedure \\
7. & Standard Arthroscopic Instrumentation \\
8. & Cosmetic procedure with faster rehabilitation \\
\end{tabular}

\section{Figures}




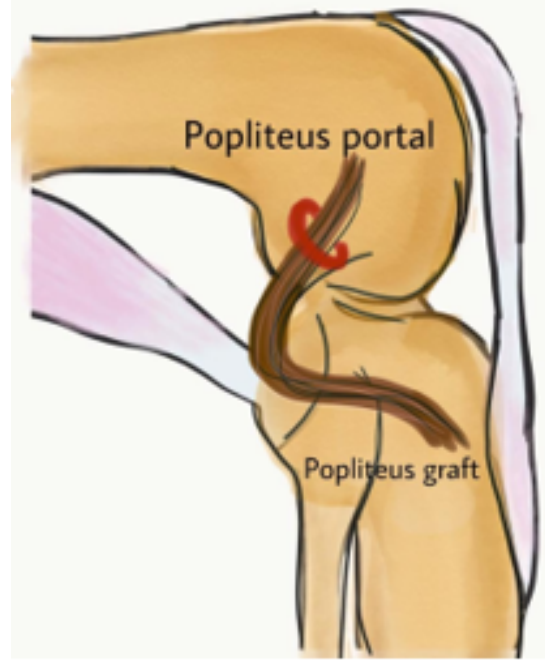

Figure 1

Location of the Popliteus portal

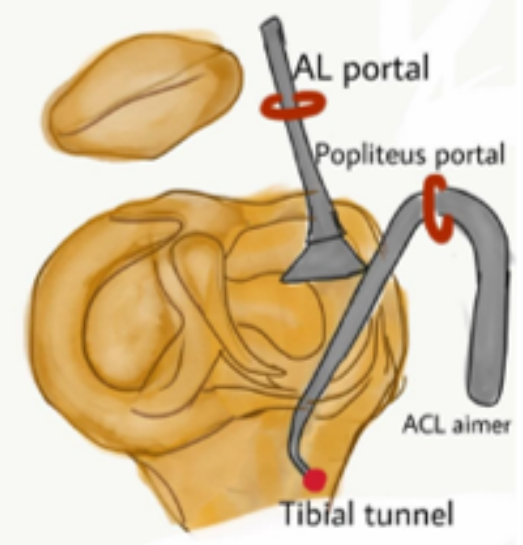

Figure 2

Location of the tibial tunnel for the popliteus graft

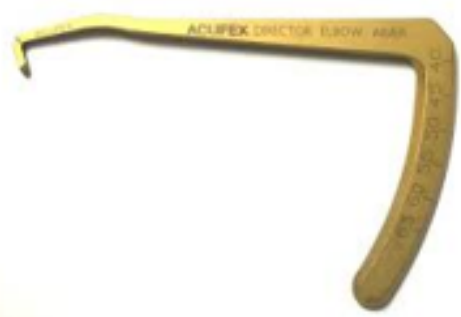

Figure 3

Acufex ACL aimer used for tibial tunnel 

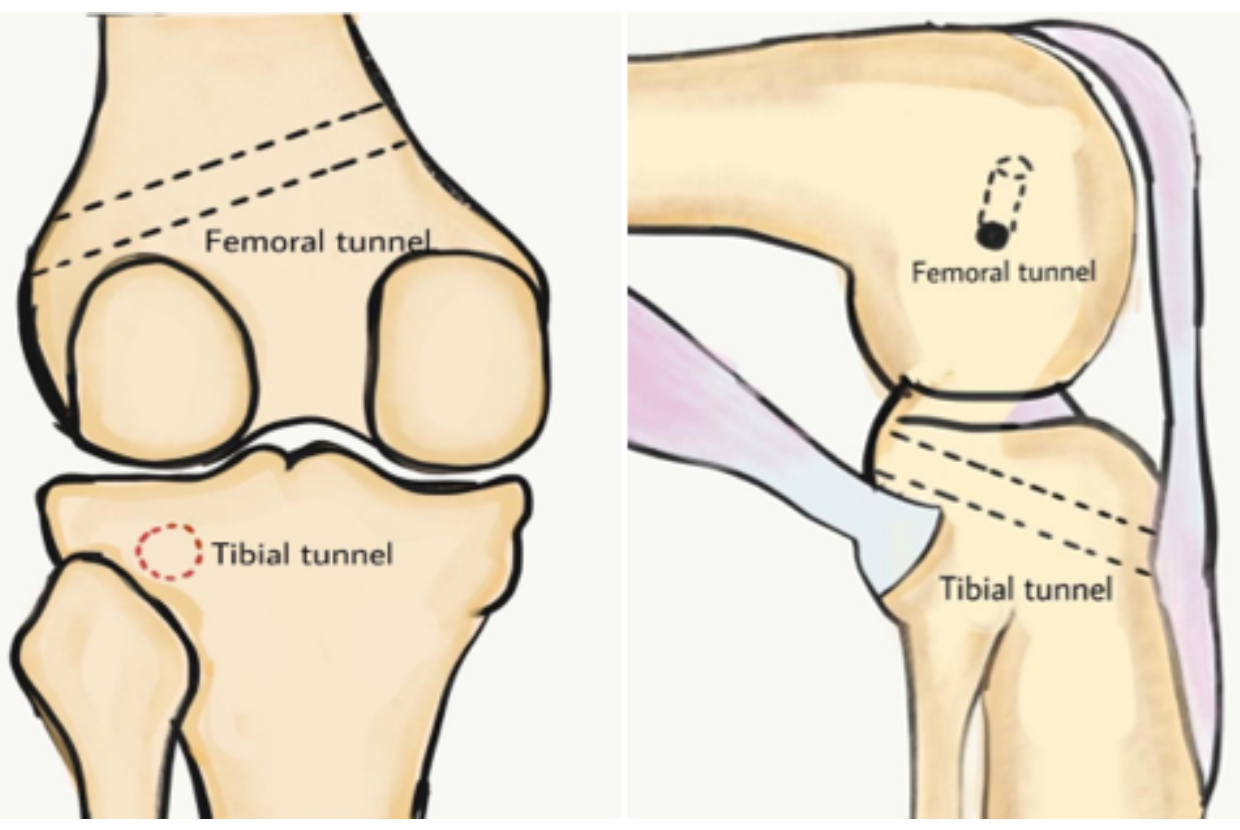

Figure 4

Diagramatic representation of Location of femoral and tibial tunnel for the popliteus graft in Anteroposterior and lateral views

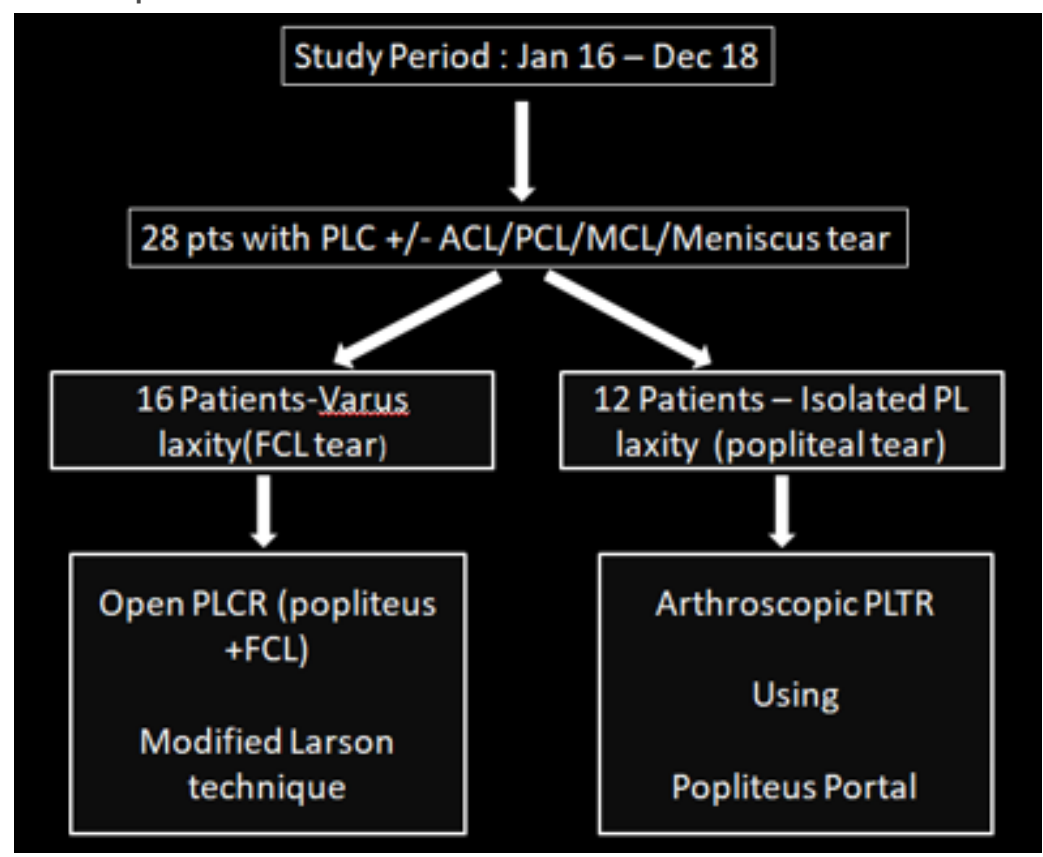

Figure 5

Brief summary of patient selection protocol 


\section{Mechanism Of Injury}

Dashboard Injury $\square$ 2W RTA

- Sports Injury $\quad$ Fall from Height

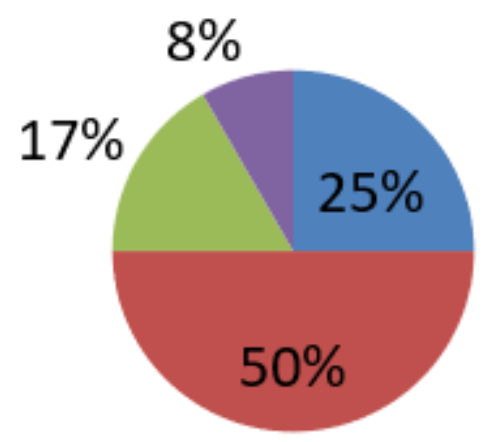

Figure 6

Mechanism of Injury

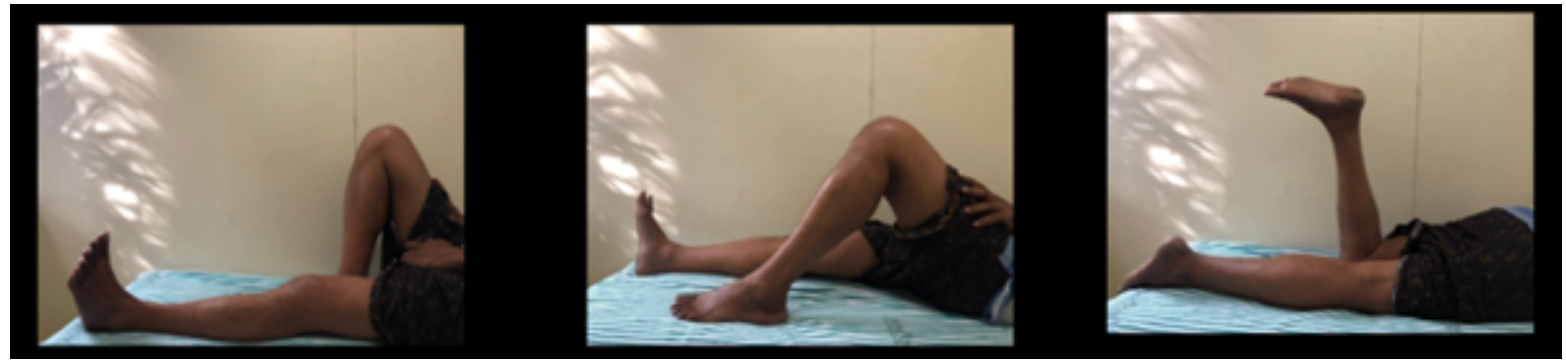

Figure 7

Clinical Photographs of one of the patients at final follow up

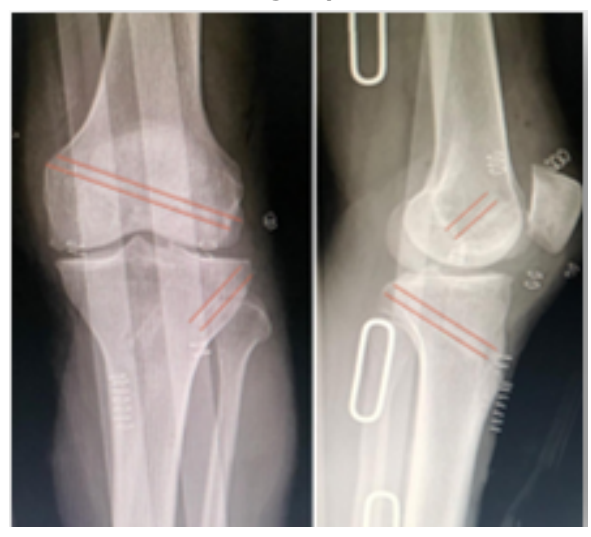

Figure 8

Post operative radiograph of one of the patients with the red lines marking the position of the femoral and tibial tunnels 


\section{IKDC Score}

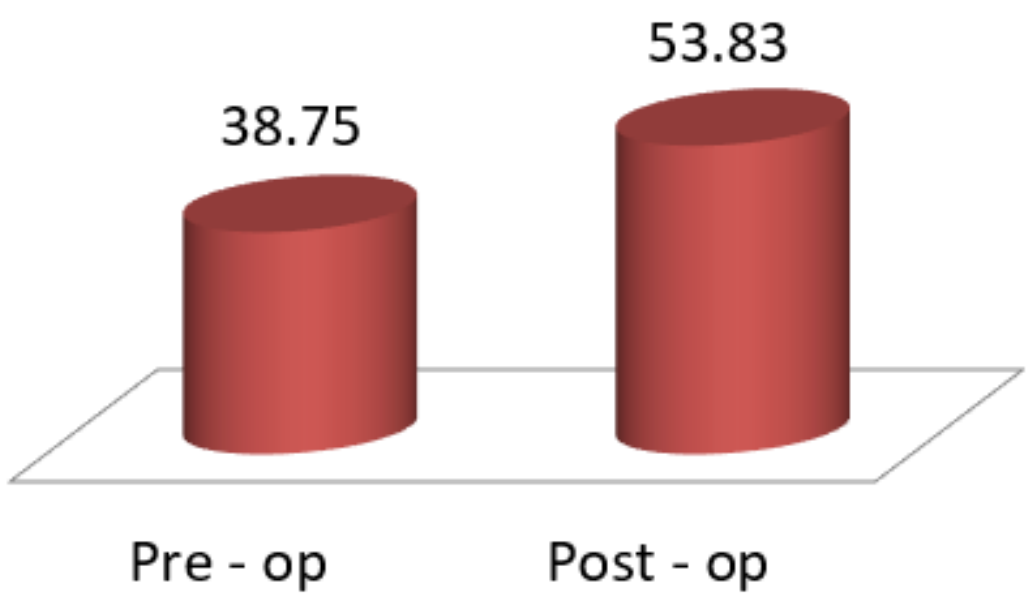

Figure 9

Graph showing the improvement of IKDC score from pre - op to post - op final follow up

\section{Tegner Activity Level}

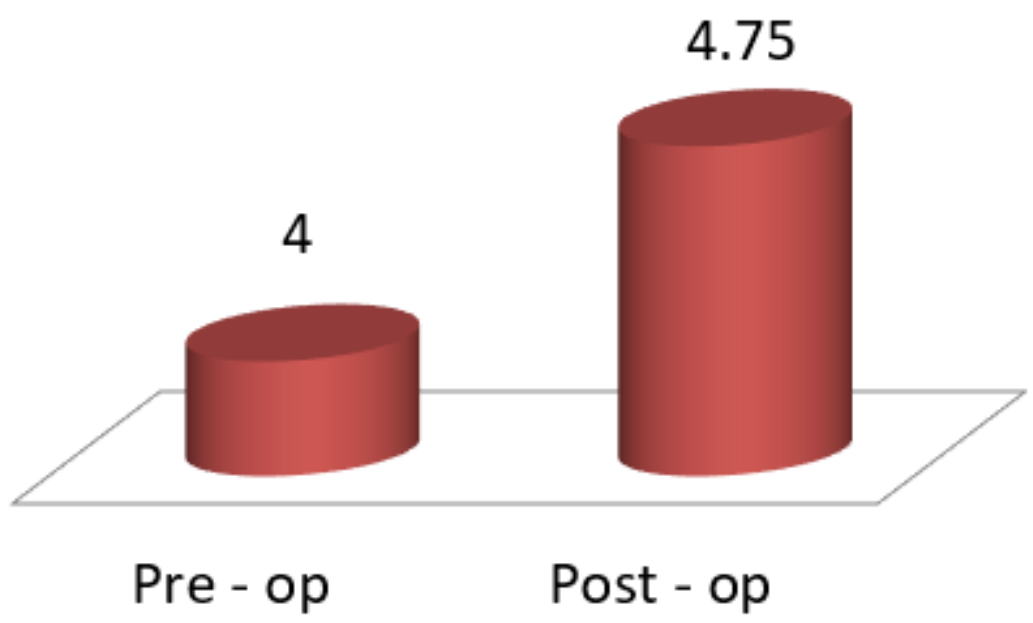

Figure 10

Graph showing the improvement of Tegner score from pre - op to post - op final follow up 